\title{
What is beautiful is useful - visual appeal and expected information quality
}

Thea van der Geest

University of Twente

T.m.vandergeest@utwente.nl

\author{
Raymond van Dongelen \\ Noordelijke Hogeschool Leeuwarden \\ Dongelen@nhl.nl
}

\begin{abstract}
Would users, when having a first glance on websites, expect that visually appealing websites contain better information than websites that are less appealing? And if they looked longer, would that change their judgment? We created two versions for 12 homepages of websites, one with low visual appeal, the other one with high visual appeal. Through a pre-test, we made sure we entered the main study with validated 'beautiful' and 'ugly' sites. In the main study, 588 participants asked to judge the expected information quality of the sites for a given task. For 11 of the 12 sites, we demonstrated that a positive judgment of the visual appeal was consistently transferred to a positive expectation of the information quality of the site, after a short exposure time. In a follow-up experiment, a week later, with 355 of the same participants, we proved that also with a longer exposure time the high visual appeal sites were expected to contain better information than the low appeal ones, although the difference between the two versions decreased somewhat. We conclude that visual appeal is an important shortcut for users to determine the information quality of a website.

Keywords: visual appeal, information quality, website quality
\end{abstract}

\section{Introduction}

Have you ever observed users who are browsing the web for information, rapidly clicking on search engine results and deciding with a glance whether to start reading the information in a site? What is the basis for that quick decision? How do users of websites judge its quality and which factors influence their decisions? Could it be their judgment about the visual appeal of the website that makes them decide that it might contain useful information?

Some theorists assume that visual appeal is a quality of a site (or any other object), determined by characteristics such as symmetry, consistency, or proportion. Others think that beauty is in the eye of the beholder, a matter of appraising the website that the user interacts with. We have chosen the judgmental approach in this study, defining beauty as the users' judgment of the attractiveness of the visible parts of a website. To stress the judgmental character we prefer to use the term visual appeal over beauty or aesthetics of the website.

Reference [1] proved that users of a website come to a judgment about the visual appeal of a website in as little as half a second, a finding that was confirmed by [2]. That initial judgment is persistent; it hardly changes when users have the chance to look longer. For all kinds of objects, and even for persons, it has been demonstrated that high visual appeal correlates with positive responses and positive expectations. In the field of HumanComputer Interaction, studies have shown effects of visual appeal on perceived usability [3,4] trust [5], and goodness [6]. Would the effect that those researchers observed also hold for the quality of the content of websites? Would users in their split second judgment expect that visually appealing websites contain better information than websites that are less appealing? And if they looked longer, would they change their judgment?

Our study focuses on the relation between visual appeal and information quality. Information is good when it is credible, correct, complete, relevant, timely, etc. But the quick decision to select a particular website from a list of search engine results can hardly be based on a careful analysis of these qualities of the content - too little time is spent with the website to judge those qualities of the information. Hence we focused on the expected information quality: The initial judgment of the usefulness of the website for informational purposes. We have asked the participants in our study to rate and select sites for their expected information quality, offering them sites with low or high visual appeal. Our study addressed the following two research questions:

1. To what extent does the visual appeal of a website influence the expected information quality of a website?

2. What is the effect of exposure time on the relation between visual appeal and expected information quality? 


\section{Manipulation check: Making sites more beautiful or more ugly}

In order to assess the effect of visual appeal on expected information quality, we had to manipulate sites to be either visually attractive or visually unattractive. To allow for a comparison of websites on visual appeal, 12 pairs of websites were created. Each pair consisted of a website with high visual appeal and a counterpart website with low visual appeal. The content of both websites was identical. The 12 pairs of websites contained information about three topics of general interest: The biography of Albert Einstein, the German isle of Ruegen as a travel destination, and medical information about headaches. For each of these three topics, four pairs of websites with high/low appeal were created.

Two graphical designers created the counterparts, guided by a set of rules for keeping the information quality constant but changing the visual appeal. For example, [7] has demonstrated that structural elements of the content, such as headings, influence the perceived information quality. Hence we formulated the rule that the designers should not add headings or re-structure the content by changing paragraphs. Previous research [8] has proved that sparse use of colors from different palettes leads to higher ratings of the visual quality of a website. Hence one of the suggestions for visual adaptation was to reduce or increase the number of colors used in the site. The graphic designers also changed visual elements such as the number of fonts used, the page grid and dividers applied, the placement and closure of illustrations, and the alignment of text. Figure 1 shows one of the pairs of websites, about Ruegen Island.

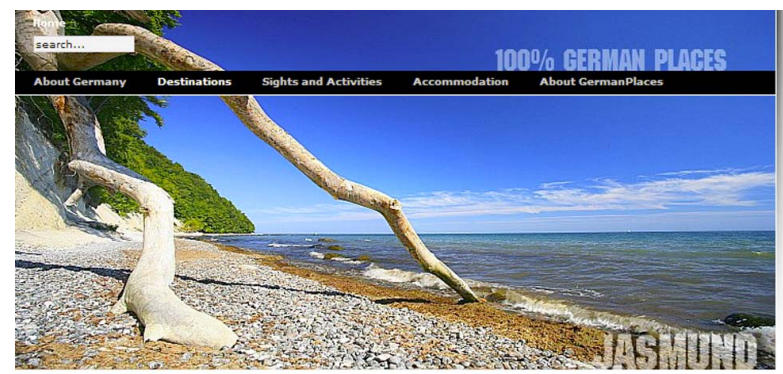

Chalk cliffs Germany in Jasmund National Park on Rugen Island on the Baltic Sea

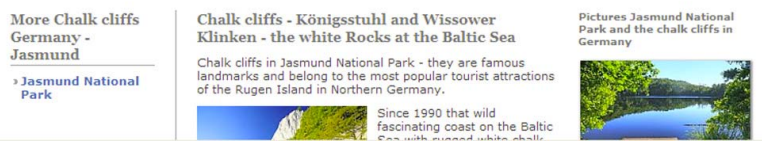

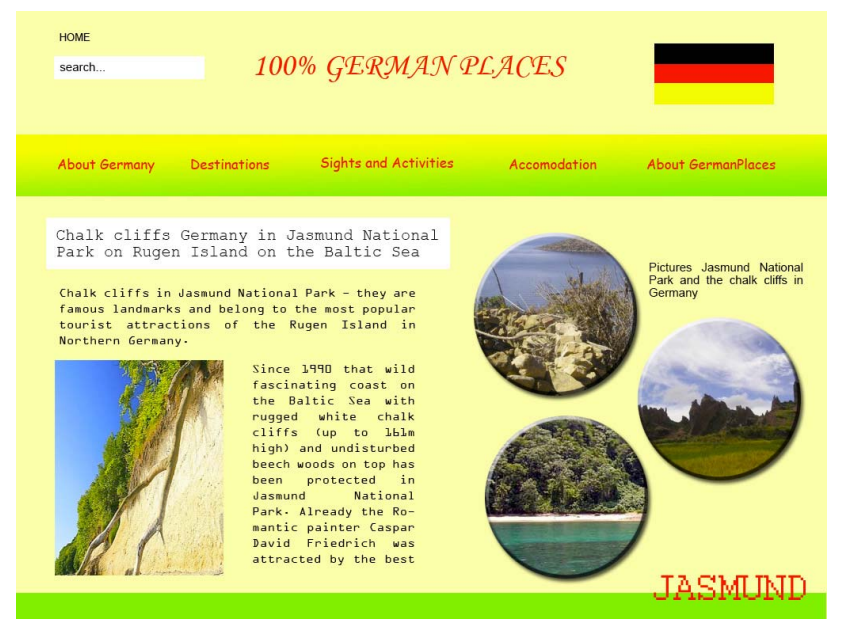

Figure 1. Pair of high-appeal (top) and low-appeal (bottom) websites

We wanted to do a manipulation check and make sure that the pairs of websites were judged differently, a point that is neglected in most previous studies of interfaces and aesthetics (e.g. in [4] and [9]). That is why we set up a pre-test in which participants rated the 24 websites. They were instructed to give their 'first impression' of each page that was presented to them, on a 7-point scale ranging from unattractive to attractive. The 24 websites were presented in random order to avoid order effects. The pages were shown for $750 \mathrm{~ms}$, a period of time that is long enough to get an impression but too short to actually read much content. The high appeal versions had to be rated significantly higher than the low appeal version ( $\mathrm{p}$ $<.05)$, in order to be useful for the main study.

In the first pre-test cycle, 78 undergraduate students of a Dutch university, the Noordelijke Hogeschool Leeuwarden, participated as part of their coursework. They all used the Internet regularly for searching information, and had no specific background in visual design. Of the 12 pairs of sites presented, ten pairs were rated significantly differently, with the high appeal version significantly more attractive than the low appeal version (all at the $\mathrm{p}<.01$ level). Two high-appeal versions of the sites on Albert Einstein were re-designed to be more distinct from their low appeal counterparts.

In a second cycle of pre-testing with 20 new undergraduate students, the 22 sites from the first pre-test and two restyled sites were presented, again in random order. The difference in attractiveness between the two restyled sites was significant $(p<.01)$, so the new sites were included in the test set. In this way, we had made sure that we would enter the main study with validated 'more beautiful' and 'more ugly' sites. It is important to note that a significant higher score on visual appeal does not necessarily mean that a site is judged beautiful - it is just more attractive than its low-appeal counterpart. Particularly, the sites about Albert Einstein did not score very high on attractiveness, whereas the sites on Ruegen 
Island were found very attractive. Since we had different numbers of male and female respondents, we checked whether there was a gender effect in both pre-test cycles; male and female participants proved to respond to the websites in the same way.

\section{Method of the main study}

In the main study, we wanted to prove to what extent visual appeal influenced expected information quality, and check whether exposure time influenced the judgment of expected information quality. To simulate the information search process, the participants were presented with three information search scenarios:

- Selection of material for a presentation about Albert Einstein (taken from [10]).

- Getting an impression of the island Ruegen as a holiday destination.

- Finding medical information about headaches (inspired by [7]).

To summarize, the study had a 3 (tasks) x 4 (sites) x 2 (appeal) research design.

The main study was conducted in two stages, but during stage one the participants were kept unaware that they would be approached for a second stage. The study was announced as aiming at getting a deeper understanding of 'first impressions' of websites. In the first session, participants filled out a questionnaire with demographic questions and questions about their Internet experience. Then they were confronted with the first task, randomly chosen from the three tasks. After having read the task description, the participant saw one version of each of the four websites, two high appeal and two low appeal versions, each for $750 \mathrm{~ms}$. The participant then judged each site on whether the information was useful and credible for their search task. Because of high correlations between the two judgments, we combined the scores in the report of the results below.

We also asked the participants in stage one to select (for each search task) four of the eight thumbnail images of the sites $(180 * 135$ pixels) as the ones they expected to have the highest information quality for their search task. This procedure was repeated for the second and third task presented. The whole session lasted an average 5 minutes per participant.

A total of 14,561 invitations were sent out for the study. The volunteering participants in the study were 588 undergraduate students of two Dutch universities (University of Twente and Noordelijke Hogeschool Leeuwarden) who were not involved in the pre-test, 247 male and 341 female, all very frequent users of the Internet. Of the participants, 114 participated in the test in a university lab setting and 474 participated in the online version of the study, taking the test at home or at a location of their choosing. Because we presented the material in two different settings, we compared the two groups for differences, but found that test location only influenced their response on one point, reported below. patterns.

The second, unannounced session took place a week later. In this session, the same three tasks were presented in a similar way, with the exception of the exposure time. This time, the participants were asked to rate four fullscreen sites for attractiveness, with a longer exposure time of 5 seconds. Of the participants of the first session, 355 participated in the second session, 152 male and 203 female, with 67 in the lab setting and 288 in the online setting. To see whether a particular group had volunteered to take part in the second part of the study, we compared the group that only took part in session 1 with those that took part in both sessions. On average, the group that only participated in the first session gave a lower rating for expected information quality than the recurring group $(\mathrm{t}$ $(6319)=-.2770, \mathrm{p}<.01)$. That is why we decided to focus the data analysis on the results of the 355 people who took part in both sessions.

\section{Effect of information quality on expected information quality}

We first analyzed the attractiveness ratings of the four websites per information search task, both when presented for a short time $(.750 \mathrm{~ms})$ and for a longer time $(5 \mathrm{sec})$. Figure 2 shows the relationship between visual appeal and the expected information quality judgment, for the two exposure times.

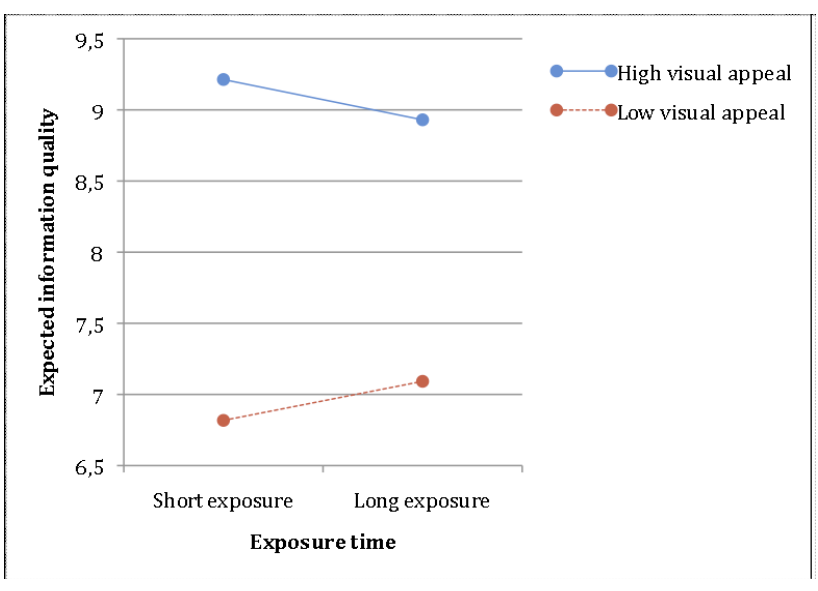

Figure 2. Mean expected information quality for high visual appeal and low visual appeal websites

As can be seen in figure 2, visually appealing sites are expected to contain better information than visually less appealing sites $(\mathrm{p}<.01)$. We proved that a positive judgment of visual appeal is consistently transferred to a positive expectation of the information quality of the site, both after a short exposure time and a longer exposure time. The difference between the two versions, that is the effect of visual appeal on expected information quality, 
decreases somewhat when the viewer has more time to scan the website $(\mathrm{p}<.01)$.

Figure 3 shows the rating for each of the website pairs. It appears that particularly for pair 2, on Albert Einstein, the participants had the same expectations for the lowappeal site as for the high-appeal site. In a next round of testing a more distinctive pair of high-appeal and lowappeal sites should replace this pair of sites. We have speculated about the possible influence of the topic (Albert Einstein) on appeal, but this study cannot give any conclusive results about the interaction between visual appeal, topic of website, and expected information quality.

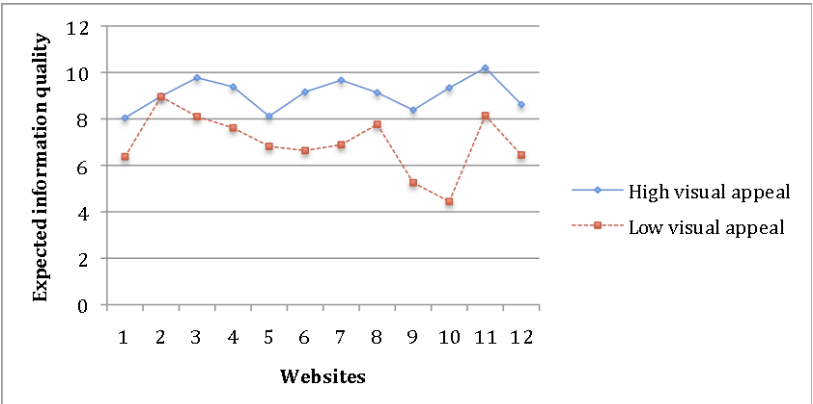

Figure 3. Mean expected information quality for each of the website pairs

In the first session, when full-screen websites were shown for a very short time $(750 \mathrm{~ms})$, eight thumbnails (four high-appeal websites and their four low-appeal counterparts) were shown to the participants. They were asked to select the four sites that they expected to have the highest information quality. The analysis focused on the question whether the high-appeal websites were selected more often than the low-appeal websites. Over all, the 588 participants selected 7104 websites, 5051 times a high-appeal site and 2053 times a low-appeal website. The effect of visual appeal on selection was analyzed and proved to be statistically significant ( $p$ $<.01)$. We screened the results for differences between the participants who took the test in the university lab and the participants who took the test online. There was a limited, but statistically significant difference between the two groups $(\mathrm{p}<.01)$. On average, the participants in the online test selected $70 \%$ of high-appeal websites, and the participants in the lab setting $75 \%$.

\section{Conclusions}

We have proven that visual appeal influences the users' perceptions of the content of a site, both when they only glance at the site and when they have more time to observe the site. Users expect more attractive sites to contain better information and select them more often for further scrutiny than less attractive sites, when conducting a search for general interest information. Since at least two of the three topics of the search tasks were serious (Einstein and headache), it appears that even sites with serious information have to draw the attention to themselves with an appealing visual design. If the site does not attract the attention and creates high expectations on its information quality, it will never be viewed in more detail and drown in the abundance of possibly informative websites that search engines yield. Visual appeal is an important shortcut for users to determine the information quality of a website. Users think that what is beautiful is useful.

\section{References}

[1] Lindgaard, G., Fernandes, G., Dudek, C., \& Brown, J. (2006). Attention web designers: You have 50 milliseconds to make a good first impression. Behaviour \& Information technology 25(2), 115-126.

[2] Tractinsky, N., Cokhavi, A., Kirschenbaum, M., \& Sharfi, T. (2006). Evaluating the consistency of immediate aesthetic perceptions of web pages. International journal of humancomputer studies, 64, 1071-1083.

[3] Schenkman, B. N., \& Jönsson, F. U. (2000). Aesthetics and preferences of webpages Behaviour \& Information technology, 19(5), 367-377.

[4] Tractinsky, N., Katz, A. S., \& Ikar, D. (2000). What is beautiful is usable. Interacting with computers, 13, 127-145.

Wirth, W., Böcking, T., \& von Pape, T. (2007). Heuristic and systematic use of search engines. Journal of computer-mediated communication, $12(3)$.

[5] Karvonen, K. (2000). The beauty of simplicity. Paper presented at the Proceedings on the 2000 conference on Universal Usability.

[6] Hassenzahl, M. (2004). The Interplay of Beauty, Goodness, and Usability in Interactive Products. Human-computer interaction, 19, 319-349.

[7] Rieh, S. Y. (2002). Judgement of information quality and cognitive authority in the web. Journal of the American society for information science and technology, 53(2), 145-161.

[8] Ivory, M.Y., \& Hearst, M. (2002). Statistical Profiles of Highly-Rated Web Sites. Paper presented at the CHI 2002 conference. Letters of the CHI 1(1), 367-374.

[9] Lavie, T., \& Tractinsky, N. (2004). Assessing Dimensions of Perceived Visual Aesthetics of Web Sites International Journal of Human-Computer Studies, 60, 269-298.

[10] Wirth, W., Böcking, T., \& von Pape, T. (2007). Heuristic and systematic use of search engines. Journal of computermediated communication, 12(3). 


\begin{abstract}
About the Authors
Dr, Thea van der Geest is an associate professor of Technical Communication at the University of Twente.

Her research and teaching are in the area of user-centered new media design, with a special interest in accessibility and affective factors determining new media adoption and use. Most of her research projects are in the context of egovernment applications and websites.
\end{abstract}

Raymond van Dongelen MSc is a lecturer at the Noordelijke Hogeschool Leeuwarden. He develops and teaches courses on The Next Web, programming and research methods. The research reported here was part of his master thesis research project. 